Original Paper

\title{
Microarray Expression Profile of Circular RNAs in Heart Tissue of Mice with Myocardial Infarction-Induced Heart Failure
}

\author{
Hong-Jin Wu Cheng-Ying Zhang ${ }^{b}$ Sai Zhang ${ }^{a}$ Min Chang ${ }^{a}$ Hong-Yun Wang ${ }^{a}$ \\ aBeijing Haidian Hospital, Haidian Section of Peking University Third Hospital, Beijing, ${ }^{\mathrm{b} T r a d i t i o n a l}$ \\ Chinese Medical Hospital of Beijing Huairou Beijing, China
}

\section{Key Words}

Myocardial infarction (MI) • Heart failure (HF) • Microarray • Noncoding RNAs • Circular RNAs

\begin{abstract}
Background/Aims: Myocardial infarction (MI) is a serious complication of atherosclerosis associated with increasing mortality attributable to heart failure. This study is aimed to assess the global changes in and characteristics of the transcriptome of circular RNAs (circRNAs) in heart tissue during MI induced heart failure (HF). Methods: Using a post-myocardial infarction (MI) model of HF in mice, we applied microarray assay to examine the transcriptome of circRNAs deregulated in the heart during HF. We confirmed the changes in circRNAs by quantitative PCR. Results: We revealed and confirmed a number of circRNAs that were deregulated during $H F$, which suggests a potential role of circRNAs in HF. Conclusions: The distinct expression patterns of circulatory circRNAs during HF indicate that circRNAs may actively respond to stress and thus serve as biomarkers of HF diagnosis and treatment.
\end{abstract}

\section{Introduction}

Despite advances in clinical and pharmacological interventions, acute myocardial infarction with subsequent left ventricular dysfunction and heart failure (HF) continues to be a major cause of morbidity and mortality worldwide [1, 2]. Approximately $25 \%$ of individuals with HF demonstrate left ventricular outflow tract (LVOT) obstruction [3]. Coronary artery occlusion leads to myocardial infarction (MI), which causes necrosis to an area of the myocardium, pathological remodeling (cardiac hypertrophy, cell death, and fibrosis), and

H.-J. Wu and C.-Y. Zhang contributed equally to this manuscript. 
cardiac dysfunction $[4,5]$. It has been accepted that HF is caused predominately by genetic variants. HF has a complex genetic basis [6, 7], and a number of biological molecules are potential biomarkers $[8,9]$ or therapeutic targets [10].To date, around 1400 mutations have been identified as being responsible for HF pathology and more than $60 \%$ of genetic variants occurred in 9 sarcomeric genes, including MYH7, MYL2 and MYBPC3 [11-14]. However, the molecular mechanisms that underlie the pathogenesis of HF remain largely unknown.

In analyses of the human transcriptome, most transcripts have little or no protein-coding capacity but rather are noncoding RNAs [15], which adds novel content to traditional protein-centric molecular biology [16]. MicroRNAs (miRNAs), a class of small noncoding RNAs, are critical in biology and medicine [17-19]. Long non-coding RNAs (lncRNAs) are involved in a variety of biological processes, such as cell-cycle control, differentiation, apoptosis, chromatin remodeling as well as epigenetic regulation [20, 21]. Circular RNAs (circRNAs) are RNA molecules with covalently joined 3'- and 5' -ends formed by back-splice events thus presenting as covalently closed continuous loops. Hence, circRNAs are characterized by scrambled exons, which were already reported more than 20 years ago [22], but mostly misinterpreted as splicing errors. Only in 2012/2013, circRNAs were rediscovered from RNA sequencing (RNA-seq) data and shown to be widespread and diverse in eukaryotic cells [2325]. CircRNAs in their proposed function as miRNAs sponges are believed to negatively regulate miRNAs, thus contributing substantially to the competing endogenous RNA netword. Therefore, circRNAs are becoming important biological molecules for understanding the mechanisms of disease and for exploring biomarkers for disease diagnosis and treatment.

In order to reveal the potential roles of circRNAs in the pathogenesis of HF, we performed microarray analysis to identify dysregulated circRNAs in heart tissue of mice with myocardial infarction induced heart failure, compared to sham groups. In the present study, we identified a number of circRNAs that are up-regulated or deregulated in heart tissue, and shown that circRNAs play roles in HF, and may represent a novel approach for HF diagnosis and treatment.

\section{Materials and Methods}

\section{Materials}

Masson's Trichrome Stain Kit was from Abcam (MA, USA); TRIZOL reagent was from Invitrogen (NY, USA). RNeasy minicolumn was from Qiagen (Valencia, CA). The GoScript Reverse Transcription System (cDNA synthesis kit) and Go Taq qPCR Master Mix (SYBR green assay) were from Promega (Madison, WI). Other chemicals and reagents were of analytical grade.

\section{Animal care}

Male C57BL/6J mice (18 - $20 \mathrm{~g}$ ) used in this study were provided by the Animal Department, Health Science Center, Peking University. All animal care and experimental protocols complied with the Animal Management Rules of the Ministry of Health of the People's Republic of China and the guide for the Care and Use of Laboratory Animals published by the US National Institutes of Health (NIH publication No. 85-23, revised 1996). All animals were housed in SPF class condition and were euthanized under sodium pentobarbital anesthesia.

\section{HF model}

The acute HF mouse model was induced by permanent ligation of the left anterior descending coronary artery, as previously described [26]. In the operation, electrocardiograms showed ST-elevated MI. mice in the sham-operated group underwent the same surgical procedure, including thread-drawing - but not ligation - at the same site.

\section{Echocardiography}

The animals were assessed via a $10 \mathrm{~S}$ phased array probe (11.5 MHz) and a Vivid 7 Dimension system (GE Healthcare Ultrasound, Horten, Norway). Eight weeks after the surgery, all the mice were anesthetized 


\section{Cellular Physiology Cell Physiol Biochem 2016;39:205-216 \\ \begin{tabular}{ll|l} 
DOI: 10.1159/000445617 & O 2016 The Author(s). Published by S. Karger AG, Basel \\
www.karger.com/cpb
\end{tabular}}

Wu et al.: Heart-failure Assoicated Circular RNA Changes in Mice Heart with Myocardial Infarction

with $10 \%$ chloral hydrate for the non-invasive examination. The parameters measured were the following: the left ventricular internal dimension at end diastole (LVIDd), interventricular septal thickness at end diastole (IVSd), left ventricular posterior wall thickness at end diastole (LVPWd), left ventricular fractional shortening (FS\%) and ejection fraction (EF\%). All parameters were measured 5 times by the same observer in a blinded manner, with the final result being the average of the 5 .

\section{Histological determination of fibrosis}

Fresh heart ventricles from the sham-operated mice $(n=10)$ and the HF mice $(n=10)$ were fixed using 4\% paraformaldehyde, dehydrated with alcohol, embedded in paraffin and cut into 5-mm-thick slices using a rotary microtome (RM2016; Leica Microsystems, Wetzlar, Germany). The slices were then stained with Masson's trichrome, and observed at x200 magnification using an inverted microscope (IX71; Olympus, Tokyo, Japan).

\section{Western blot analysis}

Proteins were prepared as described previously ${ }^{[15]}$. Freeze-clamped LV tissues (200 - $300 \mathrm{mg}$ ) were homogenized briefly in 10 volumes of lysis buffer containing (in mM) 20 Tris- $\mathrm{HCl}$ (pH, 7.4), $150 \mathrm{NaCl}, 2.5$ EDTA, $50 \mathrm{NaF}, 0.1 \mathrm{Na} 4 \mathrm{P} 207,1 \mathrm{Na}$ VV04, 1 PMSF, 1 DTT, 0.02\% (v/v) protease cocktail (Sigma-Aldrich, Missouri, USA), $1 \%(\mathrm{v} / \mathrm{v})$ Triton X-100 and $10 \%(\mathrm{v} / \mathrm{v})$ glycerol. The homogenates were centrifuged twice at $20,000 \mathrm{~g}$ at $4^{\circ} \mathrm{C}$ for $15 \mathrm{~min}$, and the supernatants were saved as total proteins. Protein concentrations were determined by the BCA method. Western blot analysis of BNP was performed by loading 20ug of total protein per well on an SDS-PAGE gel. Proteins were transferred to a PVDF membrane (Bio-Rad, California, USA), blocked with 5\% non-fat dry milk and probed with specific anti-brain natriuretic protein (BNP) (Ab19645, Abcam, USA) antibody at dilution of 1:1000 and anti-glyceraldehyde-3-phosphate dehydrogenase (GAPDH) (Ab8245, Abcam, USA) at dilution of 1:5000. The immunoreaction was visualized using HRP-conjugated goat anti-rabbit IgG secondary antibody (sc-2357, Santa Cruz Biotechnology, USA) and enhanced chemiluminescent detection kit (Amersham, London, UK), exposed to X-ray film, and quantified by densitometry with a video documentation system (Gel Doc 2000, Bio-Rad, California, USA).

\section{RNA extraction}

Ten mice each group were euthanized and hearts were quickly removed and rinsed with cold sterile phosphate buffered saline (PBS; prepared with DEPC water), about $20 \mathrm{mg}$ left ventricular tissues was collected and stored in liquid nitrogen. Five samples of about $100 \mathrm{mg}$ left ventricular tissues were collected, and total RNA was extracted by use of TRIZOL reagent (Invitrogen, NY, USA). Other heart tissues were collected, and total RNA was extracted, then cDNA for quantitative PCR analysis were synthesized from 1 ug of total RNA. Tissues were homogenized in TRIZOL reagent (Invitrogen, USA) using a Qiagen Tissuelyser. Total RNA was extracted in accordance with the manufacturer's protocol and then quantified using a NanoDrop ND-1000 spectrophotometer (Thermo Fisher Scientific, Waltham, MA). RNA integrity of each sample was assessed by denaturing agarose gel electrophoresis.

\section{Labeling and hybridization}

Sample labeling and array hybridization were performed according to the manufacturer's protocol (Arraystar, Rockville, Maryland, USA). In short, circRNAs were treated with Ribonuclease R (RNase R) to remove linear RNAs according to the manufacturer's protocol (Epicenter, Madison, WI, USA). Then, each sample was amplified and transcribed into fluorescent cRNA utilizing a random priming method with a Super RNA Labeling Kit (Arraystar). The labeled cRNAs were purified using an RNeasy Mini Kit (Qiagen, Hilden, Germany). The concentration and specific activity of the labeled cRNAs (pmol Cy3/ $\mu$ g cRNA) were measured using NanoDrop ND-1000. Then, $1 \mu \mathrm{g}$ of each labeled cRNA was fragmented by adding $5 \mu \mathrm{l} 10 \times$ Blocking Agent and $1 \mu \mathrm{l}$ of $25 \times$ Fragmentation Buffer. The mixture was heated at $60^{\circ} \mathrm{C}$ for $30 \mathrm{~min}$, and $25 \mu \mathrm{l}$ $2 \times$ Hybridization buffer was added to dilute the labeled cRNA. Then, $50 \mu \mathrm{l}$ of the hybridization solution was dispensed into the gasket slide, which was assembled with Mouse Circular RNA Array slides. The slides were incubated for $17 \mathrm{~h}$ at $65^{\circ} \mathrm{C}$ in an Agilent Hybridization Oven. The hybridized arrays were washed, fixed and scanned using an Agilent G2505C Scanner. 
Wu et al.: Heart-failure Assoicated Circular RNA Changes in Mice Heart with Myocardial Infarction

Table 1. Specific circRNAs primers for quantitative PCR analysis. PS, product size

\begin{tabular}{|c|c|c|c|c|c|}
\hline Name & Sequence & PS (bp) & Name & Sequence & PS (bp) \\
\hline GAPDH & $\begin{array}{l}\text { F:5'GTTGTCTCCTGCGACTTCA3' } \\
\text { R:5'GCCCCTCCTGTTATTATGG3' }\end{array}$ & 293 & mmu_circRNA_002690 & $\begin{array}{l}\text { F: 5' TATCTATGAGCAAGAAACTACT3' } \\
\text { R: 5' TGAGTGATCTTAAAGGATAA3' }\end{array}$ & 121 \\
\hline mmu-circRNA_CDR1as & $\begin{array}{l}\text { F:5' ACAACTGCGCAGTGTCTCCAG } \\
\text { 3'R:5'TTGGAAGACATATATCATCTG 3' }\end{array}$ & 210 & mmu_circRNA_002279 & $\begin{array}{l}\text { F: 5' ATTGTCTCTTGAAGATGAAAT3' } \\
\text { R: 5'GTCTCTCAAGTCCCATTA3' }\end{array}$ & 172 \\
\hline mmu_circRNA_001598 & $\begin{array}{l}\text { F:5'GTGGAGGAGTGGTCGGACAT3' } \\
\text { R:5'A GTAAATGGTCTGCCTGTTGG3' }\end{array}$ & 281 & mmu_circRNA_004768 & $\begin{array}{l}\text { F: 5' TATGACCAGTGTCGTATACAG3' } \\
\text { R:5' CTTAGTAGGGGGCTCCTGGCT3' }\end{array}$ & 62 \\
\hline mmu_circRNA_004775 & $\begin{array}{l}\text { F:5'TTCCTCAAGGCCCAGGCTGCTTTC3' } \\
\text { R: 5'CCATGTTCCTGACCTTCTCCAT3' }\end{array}$ & 122 & mmu_circRNA_008398 & $\begin{array}{l}\text { F:5'TGATTAAACTGGGGCTCAGACTT3' } \\
\text { R:5' CTGACGTTAGCTGGGACAGGTGG3' }\end{array}$ & 192 \\
\hline mmu_circRNA_005046 & $\begin{array}{l}\text { F:5'ACCTTGGGAAGAGAAAGAAG3' } \\
\text { R:5'CCGGCTCTTCTTTGAGTGA 3' }\end{array}$ & 155 & mmu_circRNA_008640 & $\begin{array}{l}\text { F: 5' TGTGCCTGGAGAGGATCGAGA3' } \\
\text { R: 5' GTCAGGGAGAGCTGCAGGGTT3' }\end{array}$ & 168 \\
\hline mmu_circRNA_007687 & 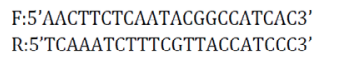 & 238 & mmu_circRNA_010147 & $\begin{array}{l}\text { F:5'CTATGCTTTCCTGCTGTTCCA3' } \\
\text { R:5'CTAGAGTAGCGTTCCACTCGTTC3' }\end{array}$ & 245 \\
\hline mmu_circRNA_013216 & $\begin{array}{l}\text { F:5'TCGATGAGTACAGGTCTAACCCTA3' } \\
\text { R:5'GATAACTCCTCCAGGCTTAGTCTAG3' }\end{array}$ & 46 & mmu_circRNA_014961 & $\begin{array}{l}\text { F:5'TGGACCTGGGCTACAGACTCTT3' } \\
\text { R:5'CTCCTGCCAGTCGATCACAAC3' }\end{array}$ & 165 \\
\hline mmu_circRNA_010567 & $\begin{array}{l}\text { F:5'TAAGCTTCAGGAAATCGAATTGGA3' } \\
\text { R:5'GGACAGACATCAACACTGTTCCA } 3\end{array}$ & 177 & Helz & $\begin{array}{l}\text { F:5'ATACTGCAGCAGCAGGAGAC3' } \\
\text { R:5'GGTGGACAGTCTTCACCCAG3' }\end{array}$ & 166 \\
\hline mmu_circRNA_015487 & $\begin{array}{l}\text { F:5'AGCTTTTCCAGTTCTGGTCA3' } \\
\text { R:5'GGAGACTTGGTCCTTCAGC3' }\end{array}$ & 127 & Sulf1 & $\begin{array}{l}\text { F:5'CTGTGCTGAGTGCTTGAGGA3' } \\
\text { R:5'GGAGAAGACCACACAGCTCC3' }\end{array}$ & 118 \\
\hline mmu_circRNA_015506 & $\begin{array}{l}\text { F:5'GGTGGCGGGAAGATGACTGT3' } \\
\text { R:5'CCGTCGTAATACTCCACGCTGT3' }\end{array}$ & 175 & Zswim6 & $\begin{array}{l}\text { F:5'TGGCACAGTGACAGAACCTG3' } \\
\text { R:5'GCTTCTGTGCAGTTGGCAAG3' }\end{array}$ & 296 \\
\hline mmu_circRNA_016128 & $\begin{array}{l}\text { F:5' CTTCTGGTTCCTGGAGTTGG3' } \\
\text { R:5' AGTTCTGGGATGCTTGCTTTA3' }\end{array}$ & 160 & $F \ln b$ & $\begin{array}{l}\text { F:5' GGGGAAAGTAACCTGCGTGA3' } \\
\text { R:5' GACATGCATTTACCGGTGCC3' }\end{array}$ & 277 \\
\hline mmu-circRNA_000174 & $\begin{array}{l}\text { F: 5' TCAGTGCCTCTCTGCTGAAGA3' } \\
\text { R: 5' 'TI'CA'TIAACT'TGGAG'I"I'IGA3' }\end{array}$ & 172 & Ubxn1 & $\begin{array}{l}\text { F:5' CTCGTCCCTGGGTCTCCC3' } \\
\text { R:5'ICTCCAGAGGC'TCA'TCCACA3' }\end{array}$ & 210 \\
\hline mmu_circRNA_001135 & $\begin{array}{l}\text { F: 5' CTTTACCATGTTTGTGACCTC3' } \\
\text { R: 5' GAACCAGGATCCAAAT'GTA3' }\end{array}$ & 138 & Zmiz1 & $\begin{array}{l}\text { F:5' CATCCCTCCACATACTCGGC3' } \\
\text { R:5' GGTGGGACCCATCT'GGAAAG3' }\end{array}$ & 193 \\
\hline mmu_circRNA_002007 & $\begin{array}{l}\text { F: 5' AGCTGGCGTGGGGGACA'TC3' } \\
\text { R: 5' GCT'T'GTAGA'TGTCAAAA'TAG3' }\end{array}$ & 71 & & & \\
\hline
\end{tabular}

Microarray and quality control

Scanned images were imported into Agilent Feature Extraction software (version 11.0.1.1) for raw data extraction. Quantile normalization of raw data and subsequent data processing were performed using the $\mathrm{R}$ software package ( $\mathrm{R}$ version 3.1.2). After quantile normalization of the raw data, low intensity filtering was performed, and circRNAs with at least 2 out of 8 samples that had flags in "P" or "M" (All Targets Value") were retained for further analyses. The log2-ratio was used for quantile normalization. CircRNAs differentially expressed between the two groups were conveniently estimated by fold-change filtering and Student's t-test. CircRNAs exhibiting fold changes $\geq 2.0$ and $p$-values $\leq 0.05$ were selected as significantly differentially expressed circRNAs. The experiment workflow is listed in Fig. 2.

Quantitative PCR Analysis

cDNA samples were prepared from total RNA of heart tissues by reverse transcription. In total, 10 up-regulated circRNAs and 10 down-regulated circRNAs were analyzed by SYBR green I dye-based detection with specific primer sequences (Table 1). The relative expression of circRNAs was determined by the $2^{\wedge(-\Delta \Delta C t)}$ method with housekeeping gene-GAPDH expression to normalize the data.

\section{Statistical analysis}

The statistical significance of microarray data was analyzed in terms of fold change using the Student's t-test and FDR was calculated to correct the P-value. FC $\geq 1.5$ and $\mathrm{P}<0.05$ were used to screen the differentially expressed circRNAs. For other statistical analysis, GraphPad Prism 5 software and Microsoft Office software were applied. Stusent's t-test was applied for comparison of two groups and differences with $\mathrm{P}<$ 0.05 were considered statistically significant.

\section{Results}

Functional evaluation of the mouse HF model

As revealed by echocardiography, HF resulted in a significant decrease contractile function as shown by percent of ejection fraction and fractional shortening (Fig. 1A). We confirmed the permanent ligation of the left anterior descending coronary artery -induced HF in mice as elevated protein level of brain natriuretic protein (BNP, a molecular marker of HF; Fig. 1B). Consistently, significant cardiac and perivascular fibrosis indicated by the staining 
Fig. 1. Functional evaluation of the mouse HF model by echocardiography, BNP pretein, and Masson's trichrome staining. (A) Representative image of echocardiography and the analysis data of percent of ejection fraction (EF\%) and fractional shortening (FS\%). (B) Representative immunoblots and averaged data for BNP protein expression. (C) Masson's trichrome staining. Values are the mean \pm SEM. ${ }^{*} p$ $<0.05,{ }^{* *} p<0.01$ vs. sham group.
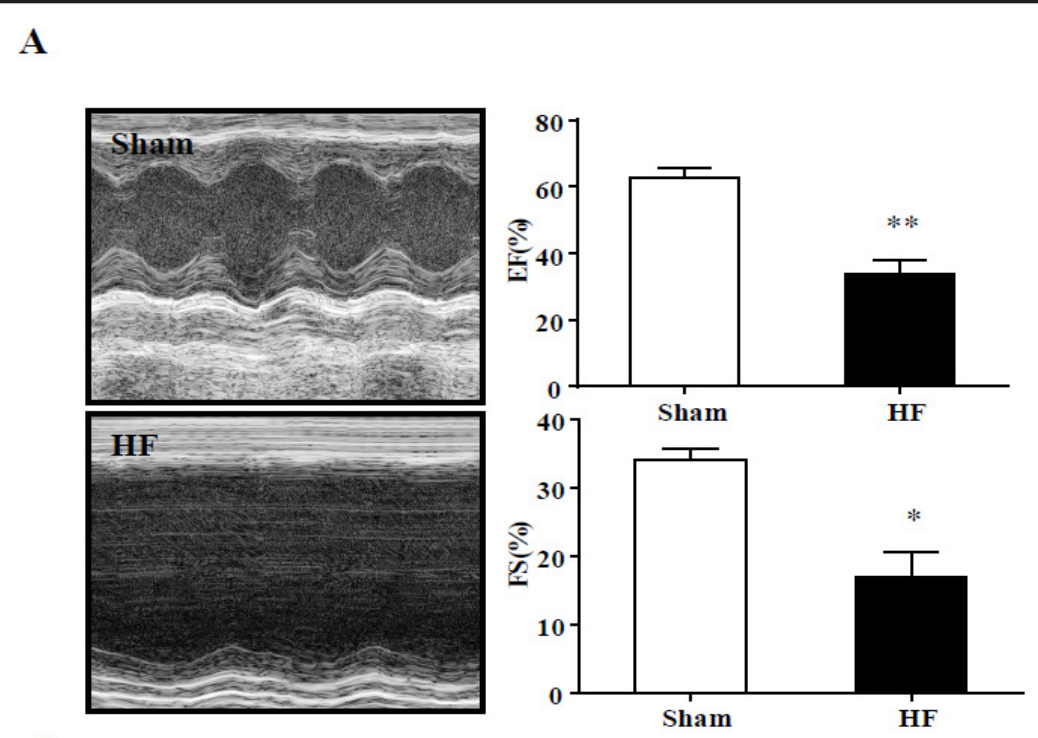

B

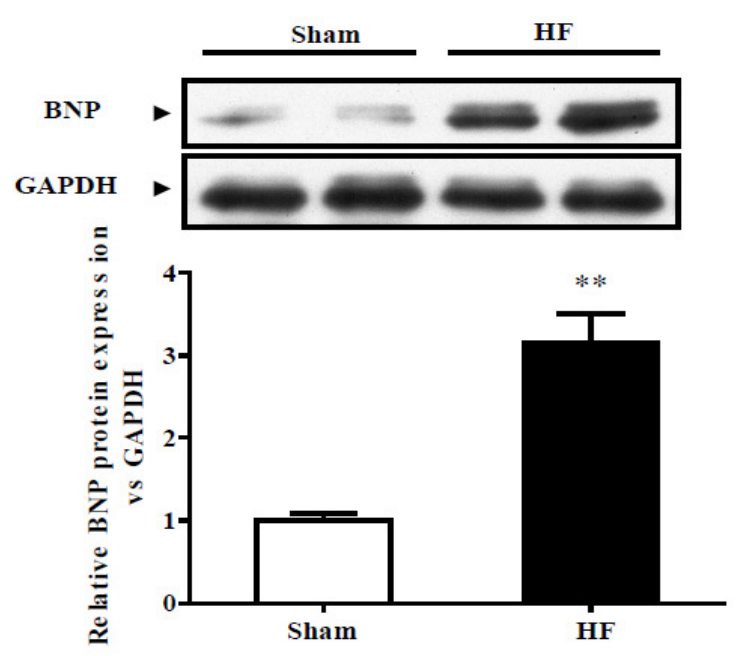

C
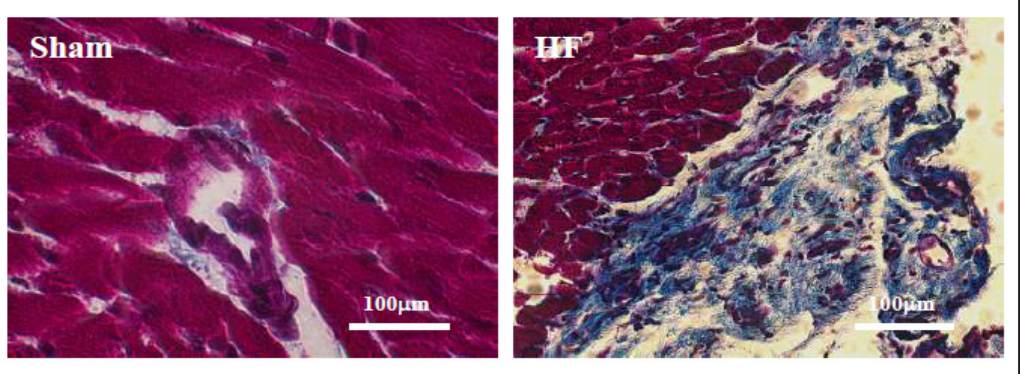

of Masson's trichrome (Fig. 1C) was observed in the HF mice compared with the sham-operated group. All these pathological modifications were linked to ventricular contractile deficiency and caused severe damage to heart function.

\section{Analysis of differentially expressed circRNAs}

In total, 1163 circRNAs were detected by Arraystar mouse circRNA Microarray. From the circRNA expression profiles, differentially expressed circRNAs were discriminated between $\mathrm{HF}$ and sham group. Hierarchical Clustering was performed to group circRNAs based on their expression levels among samples (Fig. 3A). We set a threshold as log2 fold-change $>1.5$ (Fig. 


\section{Cellular Physiology and Biochemistry \\ Wu et al.: Heart-failure Assoicated Circular RNA Changes in Mice Heart with Myocardial Infarction}

3B) and P-value $<0.05$ (Fig. 3C), and found that a total of 63 circRNAs were differentially expressed, consisting of 29 up-regulated circRNAs (Table 2) and 34 down-regulated circRNAs (Table 3). We summarized the classification of dysregulated circRNAs. Among the up-regulated circRNAs, there were 5 intergenic, 1 antisense, 1 intragnic and 22 exonic (Fig. 3D). Among the down-regulated circRNAs, there were 5 intergenic, 2 antisense, 1 intragenic and 26 exonic (Fig. 3D).

Real-time PCR validation of some differentially expressed circRNAs

We randomly selected 20 dysregulated circRNAs including 10 up-regulated circRNAs (CDR1as, 001598, 004775, 005046, 007687, 013216, 010567, 015487, 015506016128 ) and 10 down-regulated circRNAs (000174, 001135, 002007, 002690, 002279, 004768, 008398, 008640, 010147 and 014961) for verification in these myocardial tissues samples. A general consistency was shown between the real-time PCR and microarray analysis results. Six selected circRNAs in terms of up-regulated circRNAs (Fig. 4A)

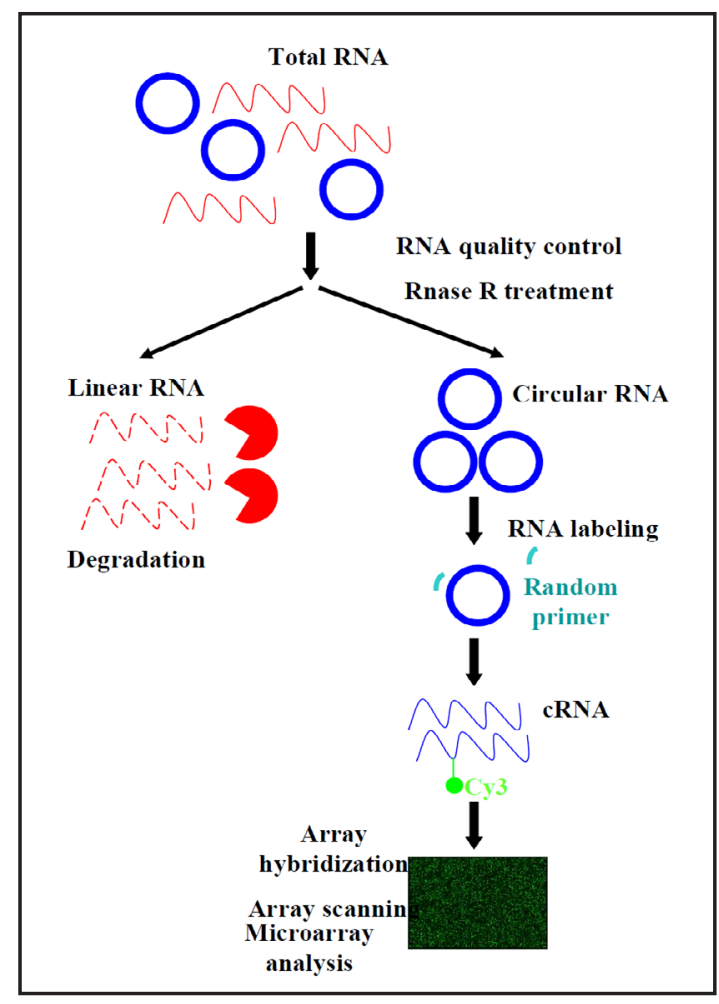

Fig. 2. Experiment workflow of microarray expression profile of circular RNAs.

and six selected circRNAs in terms of down-regulated circRNAs(Fig. 4B) were confirmed. Interestingly, circRNAs expression did not correlate with the expression of the host genes suggesting an independent regulation of transcription versus circRNA formation (Fig. 4C).

\section{Prediction of the circRNA/microRNA interaction}

Recent evidences have demonstrated that circular RNAs play a crucial role in fine tuning the level of miRNA mediated regulation of gene expression by sequestering the miRNAs. Their interaction with disease associated miRNAs indicates that circular RNAs are important for disease regulation [27-29]. To find the potential miRNA target, two confirmed circRNAs (mmu_circRNA_013216 and mmu_circRNA_010567) were selected and the circRNA/ microRNA interaction was predicted with Arraystar's home-made miRNA target prediction software based on TargetScan\& miRanda. The potential miRNA targets of mmu_circRNA_013216 include miR-181a-3p, miR-486a-5p and miR-486b-5p (Fig. 5A). For mmu_circRNA_010567, the potential miRNA targets include miR-124. miR-141 and miR-200a (Fig. 5B). It has been shown that miRNA-141 could regulate the expression level of ICAM-1 on endothelium to decrease myocardial ischemia-reperfusion injury [30], while the function of the most potential miRNAs on heart are far from clear.

\section{Discussion}

In the present study, we performed a comprehensive analysis of dysregulated circRNAs by comparing the transcriptome profiles of hypertrophied myocardial tissues with or without heart failure. A total of 1163 circRNAs were detected. We identified 29 up-regulated and 34 down-regulated circRNAs, and summarized their general characteristics. Thus, our study could provide a comprehensive understanding of circRNAs in heart failure heart and help to elucidate the molecular mechanisms of Heart failure.

\section{KARGER}


$\mathbf{A}$

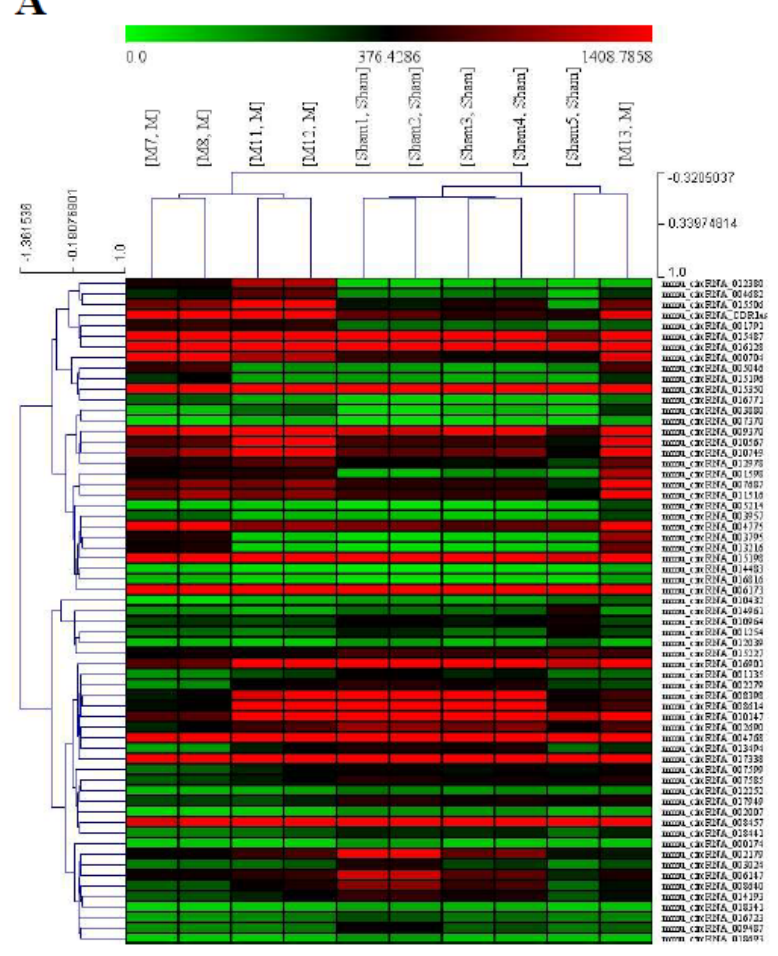

B
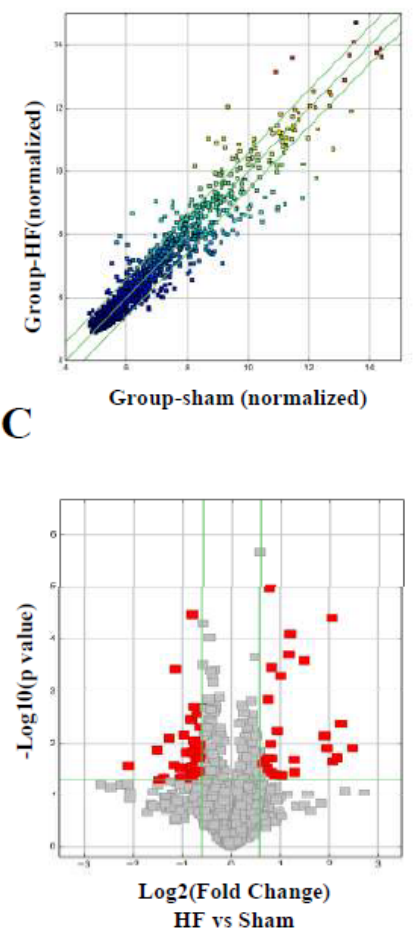

D

Up-regulation

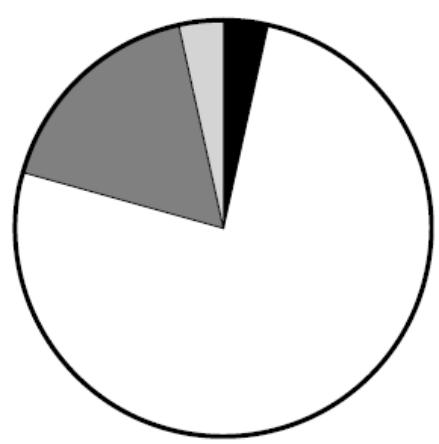

Total=29
Down-regulation

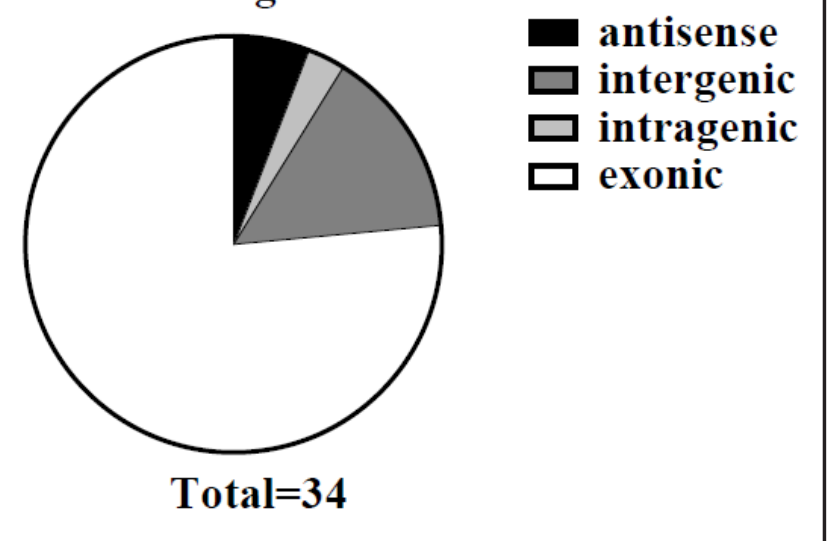

Fig. 3. Analysis of differentially expressed circRNAs. (A) The hierarchical clustering of partial differentially expressed circRNAs. 'Red' indicates high relative expression, and 'green' indicates low relative expression. (B) CircRNAs in the Scatter-Plot above the top green line and below the bottom green line indicated more than 1.5 fold change of circRNAs between the two compared samples. (C) CircRNAs (red point) in the Volcano Plots represents the 1.5 -fold up and down expressed circRNAs with statistical significance $(\mathrm{P}<0.05)$. (D) classification of dysregulated circRNAs.

Large amounts of circRNAs are recently discovered and represent a new special class of endogenous noncoding RNA. Recent researches have revealed that circRNAs are an abundant, stable, diverse and conserved class of RNA molecules [20-22]. Moreover, circRNAs can function as miRNA sponges or regulate parent gene expression to affect disease initiation and progression [27, 28, 31]. A recent report shows that circRNA ciRS-7 acts as a miR-7 sponge to suppress miR-7 activity, resulting in increased levels of miR-7 targets [29]. Other 


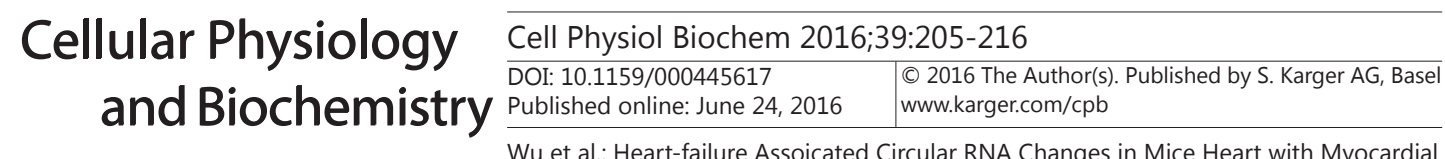

Infarction

Table 2. Up-regulated circRNAs between HF heart tissues and sham tissues. FC, fold change, FDR, false discover rate

\begin{tabular}{|c|c|c|c|c|c|c|c|c|c|}
\hline circRNA & $\mathrm{P}$-value & FDR & $\overline{F C}$ & Regulation & circRNA type & chrom & strand & best transcript & Gene Symbol \\
\hline mmu_circRNA_CDR1as & 0.000 & 0.030 & 2.816 & up & antisense & $\operatorname{chrX}$ & + & NM_001166658 & Cdr1 \\
\hline mmu_circRNA_000704 & 0.012 & 0.188 & 3.900 & up & exonic & chr7 & + & NM_011858 & Tenm4 \\
\hline mmu_circRNA_001598 & 0.000 & 0.012 & 4.196 & up & exonic & chrg & + & NM_001025600 & Cadm1 \\
\hline mmu_circRNA_001791 & 0.043 & 0.301 & 2.057 & up & exonic & $\operatorname{chr13}$ & - & NM_019930 & Ranbp9 \\
\hline $\mathrm{mmu}^{-}$circRNA-003795 & 0.020 & 0.222 & 4.484 & up & intragenic & chr1 & - & $\mathrm{NM}^{-0} 01039184$ & Cep350 \\
\hline mmu_circRNA_003880 & 0.040 & 0.291 & 2.066 & up & exonic & chr11 & + & NM_001159404 & Llgl1 \\
\hline mmu_circRNA_003957 & 0.021 & 0.226 & 1.613 & up & exonic & chr12 & + & NM_172803 & Dock4 \\
\hline mmu_circRNA_004682 & 0.035 & 0.276 & 2.470 & up & exonic & $\operatorname{chr} 18$ & - & NM_134134 & Hmgxb3 \\
\hline mmu circRNA-004775 & 0.039 & 0.289 & 1.866 & up & exonic & chr2 & - & $\mathrm{NM}^{-} 146122$ & Dennd1a \\
\hline mmu_circRNA_005046 & 0.021 & 0.226 & 2.460 & up & exonic & $\operatorname{chr} 5$ & - & NM_177047 & Auts2 \\
\hline mmu_circRNA_005214 & 0.035 & 0.276 & 1.764 & up & intragenic & chr10 & - & NR_028262 & Rmst \\
\hline mmu_circRNA_007370 & 0.030 & 0.257 & 1.567 & up & exonic & chrg & - & NM_001042752 & Neo1 \\
\hline mmu circRNA 007687 & 0.001 & 0.035 & 2.033 & up & exonic & chr11 & + & $\mathrm{NM}^{-} 198298$ & Helz \\
\hline $\mathrm{mmu}^{-}$circRNA-009370 & 0.019 & 0.222 & 1.772 & up & intragenic & $\operatorname{chr} 17$ & + & uc $0 \overline{1} 2$ ath. 2 & Rn45s \\
\hline mmu_circRNA_010567 & 0.010 & 0.171 & 1.752 & up & exonic & chr13 & - & NM_145456 & Zswim6 \\
\hline mmu_circRNA_010749 & 0.006 & 0.134 & 1.936 & up & intragenic & chr17 & + & uc0 12 ath. 2 & Rn45s \\
\hline mmu_circRNA_011516 & 0.000 & 0.006 & 1.718 & up & intronic & chr15 & - & NM_001013360 & Npcd \\
\hline mmu_circRNA_012380 & 0.012 & 0.188 & 5.577 & up & exonic & chr16 & - & NM_007938 & Epha6 \\
\hline mmu_circRNA_012978 & 0.001 & 0.080 & 1.692 & up & intronic & chr17 & - & uc00̄8awy. 1 & Tsc2 \\
\hline mmu_circRNA_013216 & 0.022 & 0.226 & 4.219 & up & intragenic & chr1 & + & NM_001198565 & Sulf1 \\
\hline mmu_circRNA_014483 & 0.030 & 0.257 & 1.671 & up & exonic & chr15 & + & NM-011767 & $\mathrm{Zfr}$ \\
\hline mmu circRNA 015196 & 0.024 & 0.239 & 1.570 & up & exonic & chr13 & - & $\mathrm{NM}^{-} 001122989$ & Cdc14b \\
\hline mmu_circRNA_015198 & 0.019 & 0.222 & 1.684 & up & exonic & $\operatorname{chr} 5$ & + & NM_175473 & Fras1 \\
\hline mmu_circRNA_015350 & 0.000 & 0.028 & 2.275 & up & exonic & chr1 & - & NM_001077695 & Ncoa2 \\
\hline mmu_circRNA_015487 & 0.004 & 0.120 & 4.748 & up & exonic & $\operatorname{chr} 7$ & + & NM_011565 & Tead2 \\
\hline mmu_circRNA_015506 & 0.007 & 0.142 & 3.770 & up & exonic & chr17 & + & NM_015800 & Crim1 \\
\hline mmu_circRNA_016128 & 0.018 & 0.221 & 4.466 & up & exonic & $\operatorname{chr} 7$ & + & NM_153591 & Nars2 \\
\hline mmu_circRNA_016771 & 0.000 & 0.016 & 2.314 & up & exonic & chr2 & - & NM_001141931 & Rbms1 \\
\hline mmu_circRNA_016816 & 0.000 & 0.032 & 1.770 & up & exonic & chr14 & + & uc007suc. 1 & Erc2 \\
\hline
\end{tabular}

Table 3. Down-regulated circRNAs between HF heart tissues and sham tissues. FC, fold change, FDR, false discover rate

\begin{tabular}{|c|c|c|c|c|c|c|c|c|c|}
\hline circRNA & P-value & FDR & FC & Regulation & circRNA_type & chrom & strand & best_transcript & $\begin{array}{l}\text { Gene } \\
\text { Symbol }\end{array}$ \\
\hline mmu_circRNA_000174 & 0.024 & 0.239 & 1.641 & down & exonic & $\operatorname{chr} 1$ & - & NM_027671 & Sntg1 \\
\hline mmu_circRNA_001135 & 0.030 & 0.257 & 1.771 & down & exonic & $\operatorname{chr} 14$ & + & NM_026283 & Samd8 \\
\hline mmu_circRNA_001254 & 0.022 & 0.226 & 1.632 & down & exonic & $\operatorname{chr} 5$ & - & uc008xwx.1 & Epha5 \\
\hline mmu_circRNA_002007 & 0.000 & 0.032 & 2.197 & down & intragenic & $\operatorname{chr} 14$ & + & NM_134080 & Flnb \\
\hline mmu_circRNA_002179 & 0.026 & 0.247 & 2.246 & down & exonic & $\operatorname{chr} 6$ & - & NM_001136065 & Hipk2 \\
\hline mmu_circRNA_002279 & 0.029 & 0.257 & 2.045 & down & exonic & $\operatorname{chrX}$ & + & NM_001034907 & $\mathrm{Zc} 3 \mathrm{~h} 12 \mathrm{~b}$ \\
\hline mmu_circRNA_002690 & 0.028 & 0.257 & 1.731 & down & exonic & $\operatorname{chr} 2$ & - & NM_172664 & Tlk1 \\
\hline mmu_circRNA_003024 & 0.036 & 0.276 & 1.652 & down & exonic & $\operatorname{chr} 18$ & - & NM_001201569 & Atp9b \\
\hline mmu_circRNA_004768 & $3 \quad 0.015$ & 0.198 & 1.884 & down & intragenic & $\operatorname{chr} 19$ & + & NM_146093 & Ubxn 1 \\
\hline mmu_circRNA_006147 & 0.043 & 0.301 & 1.734 & down & exonic & $\operatorname{chr} 5$ & - & NM_194340 & Prr14l \\
\hline mmu_circRNA_006173 & 0.002 & 0.090 & 1.679 & down & intragenic & $\operatorname{chr} 12$ & - & NM_001146176 & Max \\
\hline mmu_circRNA_007585 & 0.005 & 0.124 & 1.550 & down & exonic & $\operatorname{chr} 2$ & + & NM_001080754 & Ambra1 \\
\hline mmu_circRNA_007599 & 0.019 & 0.222 & 1.533 & down & intragenic & $\operatorname{chr} 1$ & - & NM_008551 & Mapkapk2 \\
\hline mmu_circRNA_008398 & 0.045 & 0.303 & 2.587 & down & exonic & $\operatorname{chr} 9$ & - & ENSMUST00000183955 & Mlip \\
\hline mmu_circRNA_008457 & 0.002 & 0.090 & 1.500 & down & exonic & $\operatorname{chr} 16$ & - & NM_001145886 & Tiam1 \\
\hline mmu_circRNA_008614 & 0.050 & 0.319 & 2.763 & down & exonic & $\operatorname{chr} 9$ & - & ENSMUST00000183955 & Mlip \\
\hline mmu_circRNA_008640 & 0.046 & 0.303 & 2.048 & down & exonic & $\operatorname{chr} 6$ & + & ENSMUST00000070736 & Adcyap1r1 \\
\hline mmu_circRNA_009487 & 0.003 & 0.115 & 1.752 & down & exonic & $\operatorname{chr} 6$ & - & NM_001159533 & Cacna1c \\
\hline mmu_circRNA_010147 & 0.027 & 0.250 & 4.273 & down & exonic & chr19 & - & NM_198300 & Cpeb3 \\
\hline mmu_circRNA_010432 & 0.047 & 0.306 & 1.875 & down & exonic & $\operatorname{chr} 4$ & - & NM_001081557 & Camta1 \\
\hline mmu_circRNA_010964 & 0.011 & 0.181 & 1.659 & down & intergenic & $\operatorname{chr} 1$ & + & & \\
\hline mmu_circRNA_012039 & 0.007 & 0.142 & 1.942 & down & exonic & $\operatorname{chr} 2$ & + & NM_001039939 & Asxl1 \\
\hline mmu_circRNA_012252 & 0.003 & 0.096 & 1.610 & down & exonic & $\operatorname{chr} 16$ & - & NM_175549 & Robo2 \\
\hline mmu_circRNA_013494 & 0.044 & 0.303 & 1.984 & down & intronic & $\operatorname{chr} 9$ & + & NM_011029 & Rpsa \\
\hline mmu_circRNA_014193 & 0.016 & 0.203 & 1.670 & down & exonic & $\operatorname{chr} 2$ & - & uc008nmu.1 & Rbm39 \\
\hline mmu_circRNA_014961 & 0.008 & 0.147 & 2.401 & down & exonic & $\operatorname{chr} 14$ & + & NM_183208 & Zmiz1 \\
\hline mmu_circRNA_015227 & 0.009 & 0.158 & 1.684 & down & exonic & $\operatorname{chr} 5$ & + & NM_001243123 & Fam193a \\
\hline mmu_circRNA_016723 & 0.014 & 0.193 & 1.579 & down & antisense & $\operatorname{chr} 2$ & - & NM_011701 & Vim \\
\hline mmu_circRNA_016901 & 0.014 & 0.193 & 1.703 & down & exonic & $\operatorname{chr} 17$ & + & NM_134117 & Pkdcc \\
\hline mmu_circRNA_017338 & 0.014 & 0.193 & 2.854 & down & exonic & $\operatorname{chr} 10$ & + & NM_001111065 & Reps1 \\
\hline mmu_circRNA_017949 & 0.000 & 0.012 & 1.728 & down & exonic & $\operatorname{chr} 6$ & - & NM_001081206 & Dgki \\
\hline mmu_circRNA_018341 & 0.034 & 0.274 & 1.568 & down & exonic & $\operatorname{chrX}$ & - & ENSMUST00000101616 & Smarca1 \\
\hline mmu_circRNA_018441 & 0.010 & 0.171 & 1.546 & down & antisense & $\operatorname{chr} 1$ & + & NM_201364 & BC055324 \\
\hline mmu_circRNA_018693 & 0.019 & 0.222 & 1.550 & down & exonic & $\operatorname{chr} 5$ & + & NM_198702 & Lphn3 \\
\hline
\end{tabular}

study also shows that a human circRNA CDR1as functions to bind miR-7 in neuronal tissues and sequesters away the miR-7 from its target sites [25]. In this study, we found a lot 
Fig. 4. Real-time PCR validation of some differentially expressed circRNAs and mRNA from microarray data. Twenty circRNAs were chosen for real-time PCR validation. Fold changes were calculated by the $2^{\wedge}(-\Delta \Delta \mathrm{Ct})$ method. Data shown are representative of 5 HF tissues and 5 controls; Values are the mean \pm SEM. ${ }^{*} \mathrm{p}<0.05$, $* * \mathrm{p}<0.01$ vs. sham group.

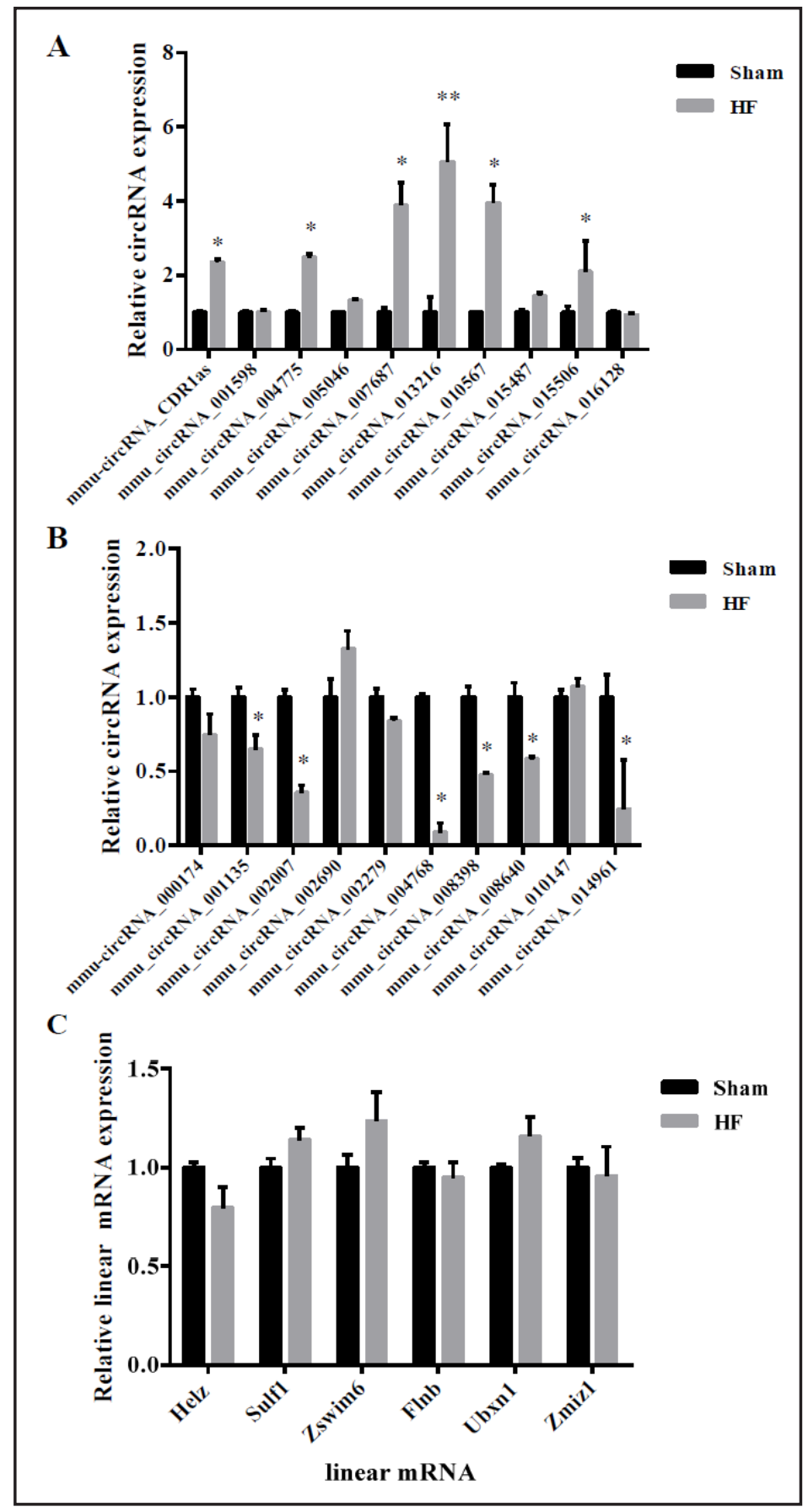

of dysregulated circRNAs in heart tissue during MI induced heart failure (HF) and predicted the circRNA/microRNA interaction with Arraystar's home-made miRNA target prediction software based on TargetScan \& miRanda. For example, among the founded potential circRNA/microRNA interactions, up-regulated circRNA mmu_circRNA_010567 is potential to be a sponge of miR-141. Moreover, miRNA-141 regulates the expression level of ICAM1 on endothelium to decrease myocardial ischemia-reperfusion injury [30]. Meanwhile, mmu_circRNA_010567 may represent a new type of mediator of heart failure occurrence and development. However, due to the limited known function of circRNAs and miRNAs, a lot of circRNA/microRNA interactions should be analyzed in the future. More importantly, by further studying the functions of circRNAs, we could improve our understanding of the 
$\mathbf{A}$

mmu_circRNA_013216vs miR-181a-3p

- Local AU _DStructure _ Postion _ Conservation Predicted By

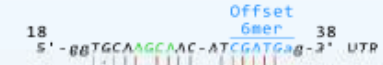

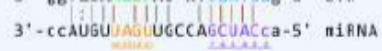

\section{mmu_circRNA_013216 vs miR-486a-5p}

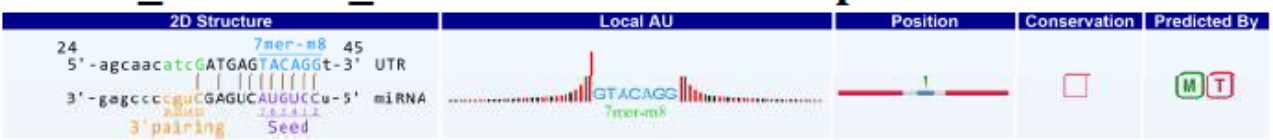

mmu_circRNA_013216 vs miR-486b-5p

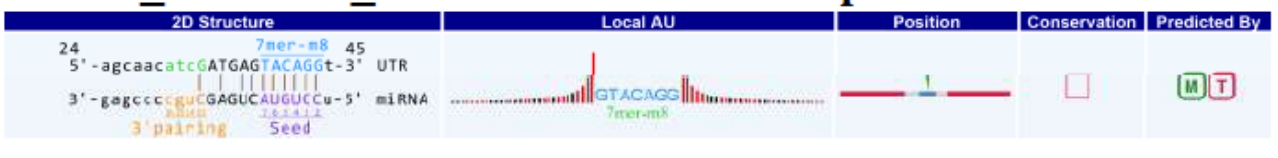

B

mmu_circRNA_010567 vs miR-124

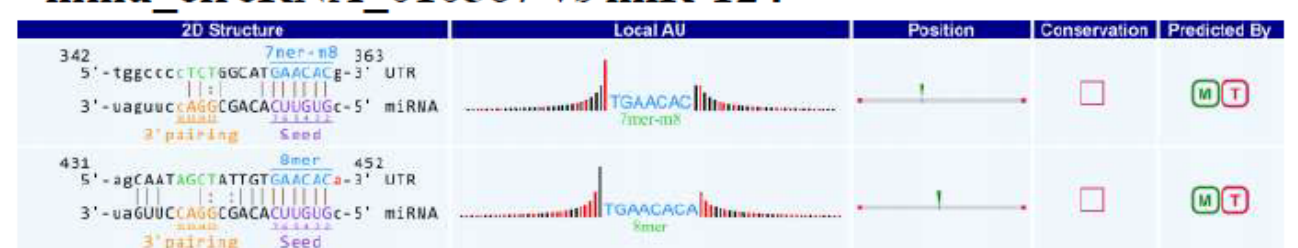

mmu_circRNA_010567 vs miR-141

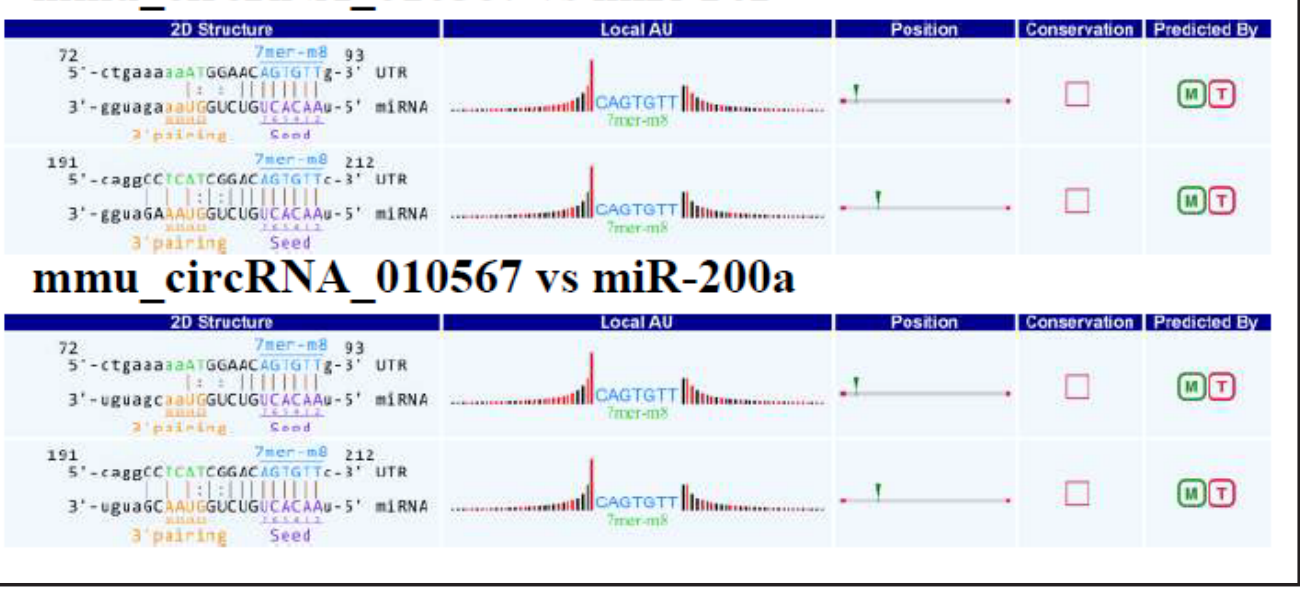

Fig. 5. A snippet of the detailed annotation for circRNA/miRNA interaction ((A) mmu_circRNA_013216; (B) mmu_circRNA_010567).

mechanisms of disease associated with circRNAs and improve the diagnosis and prevention of circRNA-associated diseases.

\section{Acknowledgments}

This project was supported by Grants from the National Natural Science Foundation of China (nos. 81273890 and 81173367).

\section{KARGER}




\section{Disclosure Statement}

The authors declare that there are no conflicts of interest.

\section{References}

1 Gerczuk PZ, Kloner RA: An update on cardioprotection: a review of the latest adjunctive therapies to limit myocardial infarction size in clinical trials. J Am Coll Cardiol 2012;59:969-978.

2 Roger VL, Go AS, Lloyd-Jones DM, Benjamin EJ, Berry JD, Borden WB, Bravata DM, Dai S, Ford ES, Fox CS, Fullerton HJ, Gillespie C, Hailpern SM, Heit JA, Howard VJ, Kissela BM, Kittner SJ, Lackland DT, Lichtman JH, Lisabeth LD, Makuc DM, Marcus GM, Marelli A, Matchar DB, Moy CS, Mozaffarian D, Mussolino ME, Nichol G, Paynter NP, Soliman EZ, Sorlie PD, Sotoodehnia N, Turan TN, Virani SS, Wong ND, Woo D, Turner MB, American Heart Association Statistics C, Stroke Statistics S: Heart disease and stroke statistics--2012 update: a report from the American Heart Association. Circulation 2012;125:e2-e220.

3 Vefali H, Manda Y, Shirani J: Myocardial viability in coronary artery chronic total occlusion. Curr Cardiol Rep 2015;17:552.

4 Hou J, Kang YJ: Regression of pathological cardiac hypertrophy: signaling pathways and therapeutic targets. Pharmacol Ther 2012;135:337-354.

5 Ladage D, Tilemann L, Ishikawa K, Correll RN, Kawase Y, Houser SR, Molkentin JD, Hajjar RJ: Inhibition of PKCalpha/beta with ruboxistaurin antagonizes heart failure in pigs after myocardial infarction injury. Circ Res 2011;109:1396-1400.

6 Creemers EE, Wilde AA, Pinto YM: Heart failure: advances through genomics. Nat Rev Genet 2011;12:357362.

7 Dorn GW, 2nd: The genomic architecture of sporadic heart failure. Circ Res 2011;108:1270-1283.

8 Maisel AS, Choudhary R: Biomarkers in acute heart failure--state of the art. Nat Rev Cardiol 2012;9:478490.

9 Wang J, Li L, Su Q Zhou Y, Chen H, Ma G, Liu T, Tang Z, Liu Y: The involvement of phosphatase and tensin homolog deleted on chromosome ten (PTEN) in the regulation of inflammation following coronary microembolization. Cell Physiol Biochem 2014;33:1963-1974.

10 Tamargo J, Lopez-Sendon J: Novel therapeutic targets for the treatment of heart failure. Nat Rev Drug Discov 2011;10:536-555.

11 Roma-Rodrigues C, Fernandes AR: Genetics of hypertrophic cardiomyopathy: advances and pitfalls in molecular diagnosis and therapy. Appl Clin Genet 2014;7:195-208.

12 Efthimiadis GK, Pagourelias ED, Gossios T, Zegkos T: Hypertrophic cardiomyopathy in 2013: Current speculations and future perspectives. World J Cardiol 2014;6:26-37.

13 Nishimura RA, Ommen SR: Hypertrophic cardiomyopathy: the search for obstruction. Circulation 2006;114:2200-2202.

14 Maron BJ, Maron MS, Semsarian C: Genetics of hypertrophic cardiomyopathy after 20 years: clinical perspectives. J Am Coll Cardiol 2012;60:705-715.

15 Bertone P, Stolc V, Royce TE, Rozowsky JS, Urban AE, Zhu X, Rinn JL, Tongprasit W, Samanta M, Weissman $\mathrm{S}$, Gerstein M, Snyder M: Global identification of human transcribed sequences with genome tiling arrays. Science 2004;306:2242-2246.

16 Schonrock N, Harvey RP, Mattick JS: Long noncoding RNAs in cardiac development and pathophysiology. Circ Res 2012;111:1349-1362.

17 Salmena L, Poliseno L, Tay Y, Kats L, Pandolfi PP: A ceRNA hypothesis: the Rosetta Stone of a hidden RNA language? Cell 2011;146:353-358.

18 Wang Y, Men M, Yang W, Zheng H, Xue S: MiR-31 Downregulation Protects Against Cardiac Ischemia/ Reperfusion Injury by Targeting Protein Kinase C Epsilon (PKCepsilon) Directly. Cell Physiol Biochem 2015;36:179-190.

19 Zhao X, Wang K, Liao Y, Zeng Q, Li Y, Hu F, Liu Y, Meng K, Qian C, Zhang Q, Guan H, Feng K, Zhou Y, Du Y, Chen Z: MicroRNA-101a inhibits cardiac fibrosis induced by hypoxia via targeting TGFbetaRI on cardiac fibroblasts. Cell Physiol Biochem 2015;35:213-226. 


\section{Cellular Physiology Cell Physiol Biochem 2016;39:205-216 \begin{tabular}{cc|c}
\cline { 2 - 2 } and Biochemistry 10.1159/000445617 & $\begin{array}{l}\text { O 2016 The Author(s). Published by S. Karger AG, Basel } \\
\text { www.karger.com/cpb }\end{array}$ \\
\cline { 2 - 3 }
\end{tabular}}

Wu et al.: Heart-failure Assoicated Circular RNA Changes in Mice Heart with Myocardial Infarction

20 Mattick JS: The genetic signatures of noncoding RNAs. PLoS Genet 2009;5:e1000459.

21 Rinn JL, Chang HY: Genome regulation by long noncoding RNAs. Annu Rev Biochem 2012;81:145-166.

22 Nigro JM, Cho KR, Fearon ER, Kern SE, Ruppert JM, Oliner JD, Kinzler KW, Vogelstein B: Scrambled exons. Cell 1991;64:607-613.

23 Salzman J, Gawad C, Wang PL, Lacayo N, Brown PO: Circular RNAs are the predominant transcript isoform from hundreds of human genes in diverse cell types. PLoS One 2012;7:e30733.

24 Jeck WR, Sorrentino JA, Wang K, Slevin MK, Burd CE, Liu J, Marzluff WF, Sharpless NE: Circular RNAs are abundant, conserved, and associated with ALU repeats. RNA 2013;19:141-157.

25 Memczak S, Jens M, Elefsinioti A, Torti F, Krueger J, Rybak A, Maier L, Mackowiak SD, Gregersen LH, Munschauer M, Loewer A, Ziebold U, Landthaler M, Kocks C, le Noble F, Rajewsky N: Circular RNAs are a large class of animal RNAs with regulatory potency. Nature 2013;495:333-338.

26 Zhuang Y, Chen X, Xu M, Zhang LY, Xiang F: Chemokine stromal cell-derived factor 1/CXCL12 increases homing of mesenchymal stem cells to injured myocardium and neovascularization following myocardial infarction. Chin Med J (Engl) 2009;122:183-187.

27 Hansen TB, Kjems J, Damgaard CK: Circular RNA and miR-7 in cancer. Cancer Res 2013;73:5609-5612.

28 Qu S, Yang X, Li X, Wang J, Gao Y, Shang R, Sun W, Dou K, Li H: Circular RNA: A new star of noncoding RNAs. Cancer Lett 2015;365:141-148.

29 Hansen TB, Jensen TI, Clausen BH, Bramsen JB, Finsen B, Damgaard CK, Kjems J: Natural RNA circles function as efficient microRNA sponges. Nature 2013;495:384-388.

30 Liu RR, Li J, Gong JY, Kuang F, Liu JY, Zhang YS, Ma QL, Song CJ, Truax AD, Gao F, Yang K, Jin BQ, Chen LH: MicroRNA-141 regulates the expression level of ICAM-1 on endothelium to decrease myocardial ischemia-reperfusion injury. Am J Physiol Heart Circ Physiol 2015;309:H1303-1313.

31 Li Z, Huang C, Bao C, Chen L, Lin M, Wang X, Zhong G, Yu B, Hu W, Dai L, Zhu P, Chang Z, Wu Q, Zhao Y, Jia Y, Xu P, Liu H, Shan G: Exon-intron circular RNAs regulate transcription in the nucleus. Nat Struct Mol Biol 2015;22:256-264. 